\title{
Homotopy perturbation and numerical solutions for MHD flow of PTT fluid through a channel embedded in a porous medium
}

\author{
B.K. Swain*, M.Das** and G.C. Dash*** \\ * Department of Mathematics, IGIT, Sarang, Dhenknal, Odisha, India. \\ **, *** Department of Mathematics, S.O.A, Deemed to be University, BBSR, Odisha, India. \\ *Corresponding Author: bharatkeshari1@gmail.com
}

Submitted :25/10/2020

Revised :26/12/2020

Accepted :03/01/2021

\begin{abstract}
An analysis is made of the steady one dimensional flow and heat transfer of an incompressible viscoelastic electrically conducting fluid (PTT model) in a channel embedded in a saturated porous medium. The pressure driven flow is subjected to a transverse magnetic field of constant magnetic induction (field strength). The heat transfer accounts for the viscous dissipation. The governing equation (a nonlinear ordinary differential equation) is solved analytically (Homotopy Perturbation Method) and numerically (Runge-Kutta method with shooting technique) providing the consistency of the result. The role of Deborah number substantiates both Newtonian and non-Newtonian aspects of the flow model. The inclusion of two body forces affects rheological property of the flow model considered. Temperature distribution in the boundary layer is shown when the channel surfaces are held at constant temperatures. A novel result of the analysis is that the contribution of viscous dissipation is found to be negligible as the variation of temperature is almost linear across the flow field in the present PTT fluid model indicating preservation of thermal energy loss.
\end{abstract}

Keywords: MHD; PTT; Homotopy perturbation method; Porous medium; Heat transfer.

\section{Nomenclature}

$B$ Magnetic flux

$\boldsymbol{\nu}_{0}$ constant flux density

$\mathrm{D} / \mathrm{Dt}$, material time derivative

$\mathrm{Kp}$ porosity parameter

L characteristic length

$\mathrm{M}$ magnetic parameter
De Deborah number,

$J$ electric current density

I identity tensor

p pressure

$T^{\prime \prime}$ Cauchy stress tensor

$V$ velocity vector 


\section{Greek Symbol:}

\author{
$\vec{\tau}$ extra stress tensor \\ $\Lambda$ relaxation time \\ $\mu$ constant viscosity coefficient \\ $\sigma$ electrical conductivity
}

\author{
$\rho$ fluid density \\ $\varepsilon$ elongation parameter \\ $v_{\text {gradient operator }}$
}

\section{INTRODUCTION}

Many industrial or common fluids with complex structure such as polymeric fluids like molten plastics, paint, blood, egg whites, foams, and granular media display unusual behavior other than simply interplay of inertial and viscous forces as in case of Newtonian fluid. The present study mainly applied to flows of polymeric fluid due to presence of long chain molecules. The study of such fluids falls in the field of viscoelasticity, due to display of both viscous and elastic behaviors. The linear affine Phan-Thien-Tanner (PTT) model is to be used out of many mathematical models of viscoelastic fluid like PTT model and can be applied to flows of polymer melts. The flow of $2.5 \%$ polyisobutylene solutions, fitting the PTT model well with the model parameter $\lambda=0.8$ and a typical value $\lambda=0.25$, is suggested for high density polyethylene melts.

For the PTT model parameter $\lambda$, values lie in the range $\lambda \in[0,1]$, with the values approaching 1 being unrealistically large. One of the best known applications in this area was the disposal of red mud (the waste product of the bauxite alumina). The result of the work was carried out by Nguyen and Boger implemented by ALCOS of Australia to reduce environmental impact of their waste disposal system (Nguyen \& Boger, 1998). Boger also applied this result to oil industry to maintain the fluidity of high wax content crude oil. Further, the techniques have been used to pipeline transportation, inkjet printing, and delivery of agricultural chemical. The constitutive equations proposed by researchers (Phan-Thien and Tanner, 1977, Tanner, 2000\&Oliveira and Pinho, 1999) derived the analytical expression for velocity field and stress components of the fully developed flow of PTT fluids through channel and pipe.

The following recent contributions are noteworthy also. Stability analysis of constitutive equations for polymer melts in viscoelastic flows was studied by Grillet et al. (Grillet et al., 2002). For the PTT model equations, the instabilities are predicted for both plane coquette and Poiseuille flows using transient finite element calculations. They have observed that ballooning of the continuous spectrum, which can cause spurious instability, is significantly stabilized for PTT or upper convected Maxwell model constitutive equations. Moreover, Grillet et al. here discussed the stability analysis of constitutive equations of polymer melts, representative of PTT viscoelastic model in viscometric flows. Numerical study of slip effects on PTT fluids in duct flow have been reported by Oveisi and Abdollahzadeh (Oveisi \& Abdollahzadeh, 2016). Shah et al. (Shah et al., 2017) have presented the exact solution for PTT fluid on a vertical moving belt for lift with slip condition. The interesting finding is that the velocity of PTT fluid increased with the incorporation of slip condition on vertical belt. Further, Ferras et al. (Ferras et al., 2020) have addressed the problem of Newtonian and viscoelastic fluid (PTT) fluid flows through an abrupt 1:4 expansion with slip boundary conditions. Another interesting problem is shear thinning and elasticity in flow around a sphere in a cylindrical tube. Their work considers purely viscous and viscoelasticity type and is of practical interest (Song et al., 2010). To investigate the effect of shear thinning and elasticity, four representative constitutive equations are considered, that is, Newtonian, Carreau, Oldroyd B, and PTT models. It was found that both shear thinning and elasticity lead to a decrease in the drag coefficient. 
Jamalbadi and Oveisi (Jamalabadi \& Oveisi, 2016) have considered PTT fluid model of viscoelastic fluid flow around a cylinder in a duct. Numerical solutions are obtained for the $2 \mathrm{D}$ viscoelastic flow. Finite element scheme is implemented. Recently Hussain et al. (Hussain et al. 2019) have studied peristaltic flow of PTT fluid in a flexible cylindrical tube. The core layer (inner) layer fluid satisfies the constitutive equations of PTT fluid model, and peripheral (outer) layer is known as a Newtonian fluid.

All the above works are only confined to flow without considering heat transfer in the flow domain. The following recent works take care of heat transfer phenomenon. Khan \& Tlili (2020) studied the significance of activation of microorganisms. Al-Khaled \& Khan (2020) have studied the thermal aspects of Cassonnano liquid. Ahmed et al. (2020) have reported novel micro structure features on a curved channel. In addition to the above works, the work of Khan et al. (2020) on thixotropic nano-liquid configured by Riga surface with gyrotactic microorganism and activation energy attracted the attention and interest in the field of biotechnology applications. Another work which includes heat and mass transfer characteristics in flow of bi-viscosity flow through a curved channel with contracting and expanding walls has been reported by Ahmed et al. (2020).

The works discussed above are confined to electrically nonconducting flow without porous medium. Further, on careful stud, it is revealed that the combined effects of applied transverse magnetic field (a force-acting-atdistance) and permeability of the saturated porous medium, an inbuilt body force of the medium, on PTT fluid model of viscoelastic fluids have not been discussed in any of the above reported works. Moreover, solution methods in most of the papers reflected above are numerical, but, in the present paper, both analytical (homotopy perturbation) and numerical methods ( $4^{\text {th }}$ order Runge-Kutta method with shooting technique) have been applied to solve the governing equations with prescribed boundary conditions. The analysis presents a good agreement of the solutions by two methods which assert the accuracy of the results reported herein.

The following assumptions are made during the course of the present analysis. Some are realistic and pertaining to polymeric processing and other industrial applications are oriented to act as a coolant/heating element.

i. The flow is incompressible and has constant physical properties.

ii. We assume that the magnetic Reynolds number is so small that the induced magnetic field can be neglected in comparison with the applied one.

iii. The Joule heating has been neglected as the study is limited to low magnetic Reynolds number.

iv. The Darcy dissipation is neglected because present study is not of extreme size or at low temperature or in high gravity field.

v. It is also assumed that no applied and polarization voltage exist. This then corresponds to the case when no energy is added to or extracted from the fluid by the electric field.

vi. Further, it is assumed that the porous medium is uniform and fully saturated.

\section{MATHEMATICAL FORMULATION}

The constitutive equation of an incompressible, PTT fluid (Phan-Thien and Tanner, 1977, \&Tanner, 2000) is of the form

$\overrightarrow{T^{*}}=-p I+\vec{\tau}$,

$f(\operatorname{tr}(\vec{\tau})) \vec{\tau}+\lambda \vec{\tau}=2 \mu A_{1}$ 
$\nabla$

where $A_{1}$ is the first Rivlin-Ericksen tensor and $\tau$ is Oldroyd upper convected derivative defined as

$$
\begin{aligned}
& A_{1}=\nabla \vec{V}+(\nabla \vec{V})^{T}, \\
& \nabla=\frac{D \vec{V}}{D t}-\vec{\tau} \cdot \nabla \vec{V}-\nabla \vec{V}^{T} \cdot \vec{\tau} .
\end{aligned}
$$

$\vec{V}$ and $\nabla \vec{V}$ are defined in two dimension as follows:

$$
\vec{V}=\left(\begin{array}{l}
u(x, y) \\
v(x, y)
\end{array}\right), \nabla \vec{V}=\left(\begin{array}{ll}
\frac{\partial u}{\partial x} & \frac{\partial u}{\partial y} \\
\frac{\partial v}{\partial x} & \frac{\partial v}{\partial y}
\end{array}\right)
$$

In the present case

$$
\vec{V}=\left(\begin{array}{c}
u(y) \\
0
\end{array}\right) \text { and } \nabla \vec{V}=\left(\begin{array}{cc}
0 & \frac{\partial u}{\partial y} \\
0 & 0
\end{array}\right)
$$

Two forms of the PTT models are in common use, where the function $f$ is defined as follows:

$$
\begin{aligned}
& f(\operatorname{tr}(\vec{\tau}))=1+\frac{\varepsilon \lambda}{\mu} \operatorname{tr}(\vec{\tau}), \text { linear form (Phan-Thien and Tanner, 1977) } \\
& f(\operatorname{tr}(\vec{\tau}))=\exp \left(\frac{\varepsilon \lambda}{\mu} \operatorname{tr}(\vec{\tau})\right), \text { exponential form (Tanner, 2000). }
\end{aligned}
$$

When $\varepsilon$ tends to be zero and the trace of the stress tensor is small, then (2.4) and (2.5) become upper convected Maxwell (UCM) model.

The field equation of MHD flow becomes

$$
\begin{aligned}
& \rho \frac{D \vec{V}}{D t}=\nabla \cdot \overrightarrow{T^{*}}+\vec{J} \times \vec{B} . \\
& \overrightarrow{T^{*}}=-p I+\vec{\tau}
\end{aligned}
$$


where $\vec{\tau}=\left(\begin{array}{ll}\tau_{x x} & \tau_{x y} \\ \tau_{y x} & \tau_{y y}\end{array}\right)$, the extra stress tensor, and $\overrightarrow{T^{*}}$ is the total stress tensor.

The continuity equation is given by

$\operatorname{tr} A_{1}=0$

For the present problem, the stress tensor and velocity field turn into the form

$\vec{V}=u(y) \hat{i}, \vec{\tau}=\vec{\tau}(y) \hat{i}$

where $\hat{i}$ is the unit vectors and $\mathrm{u}(\mathrm{y})$ is the velocity in the $\mathrm{x}$-direction, respectively.

Suppose that external electric field is negligible and the magnetic Reynolds number is very small. Therefore, the MHD body force can be considered as

$\vec{J} \times \vec{B}=-\sigma B_{0}^{2} \vec{V}$

The continuity equation is satisfied by the assumptions in (2.8).

The equation of motion (2.6) gives the following equations:

$-\frac{\partial p}{\partial x}+\frac{\partial \tau_{x y}}{\partial y}-\sigma B_{0}^{2} u-\frac{\rho v u}{K_{p}}=0$

$-\frac{\partial p}{\partial y}+\frac{\partial \tau_{y y}}{\partial y}=0$

$\frac{-\partial p}{\partial z}=0$

Substituting (2.8) in (2.1) and (2.2), we get

$f(\operatorname{tr}(\tau)) \tau_{x x}-2 \lambda \tau_{x y} \frac{d u}{d y}=0$

$f(\operatorname{tr}(\tau)) \tau_{x y}-\lambda \tau_{y y} \frac{d u}{d y}=\mu \frac{d u}{d y}$ 
$f(\operatorname{tr}(\tau)) \tau_{y y}=0$

Applying the linear form of $f$, we get

$\tau_{y y}=0, \tau_{x x}=2 \frac{\lambda}{\mu} \tau_{x y}^{2}$

Substituting the following nondimensional variables and parameters

$x^{*}=\frac{x}{L}, y^{*}=\frac{y}{L}, u^{*}=\frac{u}{U}, \tau^{*}=\frac{\tau L}{\mu U}, p^{*}=\frac{p L}{\mu U}$,

$M^{*}=\frac{\sigma B_{0}^{2} L^{2}}{\mu}, K_{p}^{*}=\frac{K_{p}}{L^{2}}, D_{e}=\frac{\lambda U}{L}$

in (2.10), we get (after dropping the asterisks)

$-\frac{\partial p}{\partial x}+\frac{\partial \tau_{x y}}{\partial y}-N u=0$, where $N=M+\frac{1}{K_{p}}$

Now, putting (2.16) in (2.4), we have

$$
\left(1+2 \varepsilon D_{e}^{2} \tau_{x y}^{2}\right) \tau_{x y}=\frac{d u}{d y}
$$

Since $\frac{d p}{d y}=0$, from (2.18) and (2.19), we get

$\frac{d^{2} \tau_{x y}}{d y^{2}}-\left(M+\frac{1}{K_{p}}\right)\left(\tau_{x y}+2 \varepsilon D_{e}^{2} N \tau_{x y}^{3}\right)=0$

The suitable no-slip boundary conditions are as follows:

$u(0)=0, u(1)=0 \Rightarrow \frac{d}{d y} \tau_{x y}(0)=\frac{d}{d y} \tau_{x y}(1)=P$,

where $\mathrm{P}$ is constant pressure gradient. 
For brevity, we introduce $\psi=\tau_{x y}, N=M+\frac{1}{K_{p}}, \beta=2 \varepsilon D_{e}^{2}\left(M+\frac{1}{K_{p}}\right)$

and we get

$\frac{d^{2} \psi}{d y^{2}}=N \psi+\beta \psi^{3}$

$\psi^{\prime}(0)=\psi^{\prime}(1)=P$

where' denotes derivative with respect to $\mathrm{y}$.

The equation (2.22) presents a nonlinear oscillatory system.

(i) When $\mathrm{N}<0$ and $\beta<0$, equation represents a hard spring system, and the motion of a mass is oscillatory.

(ii) When $\mathrm{N}<0$ and $\beta>0$, it represents a soft spring system, and the motion of a mass appears to be nonoscillatory. However, the qualitative analysis is required for complete analysis (Dennis, 2009).

However, in case of Equation (2.22), both cases do not arise as the model is related to a fluid flow, and here $\mathrm{N}$ and $\beta$ as defined are always positive.

\section{SOLUTION OF THE PROBLEM}

We set (2.22) into following form by introducing ' $\mathrm{q}$ ' as the homotopy perturbation parameter (He, 2005), and we get

$$
\begin{aligned}
& (1-q)\left\{\psi^{\prime \prime}-N \psi\right\}+q\left\{\psi^{\prime \prime}-N \psi-\beta \psi^{3}\right\}=0, \\
& \psi^{\prime}(0)=\psi^{\prime}(1)=P
\end{aligned}
$$

Now, putting

$\psi=\psi_{0}+\psi_{1} q+\psi_{2} q^{2}+\ldots \ldots \ldots .$. in (3.1) and (3.2) and collecting the coefficients of $\mathrm{q}^{0}$ and $\mathrm{q}^{1}$, we get

(i) $\quad \psi_{0}^{\prime \prime}-N \psi_{0}=0$,

$$
\psi_{0}^{\prime}(0)=P, \psi_{0}^{\prime}(1)=P
$$


(ii)

$$
\begin{aligned}
& \psi_{1}^{\prime \prime}-N \psi_{1}=\beta \psi_{0}^{3}, \\
& \psi_{1}^{\prime}(0)=0, \psi_{1}^{\prime}(1)=0 .
\end{aligned}
$$

Solving (3.3) and (3.5) with the boundary conditions (3.4) and (3.6), respectively, we get

$$
\begin{aligned}
& \psi_{0}=c_{1} e^{\sqrt{N} y}+c_{2} e^{-\sqrt{N} y} \\
& \psi_{1}=c_{3} e^{\sqrt{N} y}+c_{4} e^{-\sqrt{N} y}+\beta\left\{\frac{c_{1}{ }^{3} e^{3 \sqrt{N} y}}{8 N}+\frac{c_{2}{ }^{3} e^{-3 \sqrt{N} y}}{8 N}+\frac{3 c_{1}{ }^{2} c_{2} e^{\sqrt{N} y}}{2 \sqrt{N}} y-\frac{3 c_{1} c_{2}{ }^{2} e^{-\sqrt{N} y}}{2 \sqrt{N}} y\right\}
\end{aligned}
$$

The HPM iterative process is considered up to first order. Hence,

$$
\psi=\lim _{q \rightarrow 1}\left(\psi_{0}+\psi_{1} q\right)
$$

The constant of coefficients can be calculated using boundary conditions.

$$
\begin{aligned}
& c_{1}=\frac{P}{\sqrt{N}\left(e^{-\sqrt{N}}-e^{\sqrt{N}}\right)}\left(e^{-\sqrt{N}}-1\right), c_{2}=c_{1}-\frac{P}{\sqrt{N}}, c_{3}=c_{4}+c_{6}, c_{4}=\frac{c_{6} e^{\sqrt{N}}-c_{8}}{e^{-\sqrt{N}}-e^{\sqrt{N}}}, \\
& c_{5}=\beta\left\{\frac{3 c_{1}^{3}}{8 \sqrt{N}}-\frac{3 c_{2}{ }^{3}}{8 \sqrt{N}}+\frac{3 c_{1}{ }^{2} c_{2}}{2 \sqrt{N}}-\frac{3 c_{1} c_{2}{ }^{2}}{2 \sqrt{N}}\right\}, c_{6}=-\frac{c_{5}}{\sqrt{N}}, c_{8}=-\frac{c_{7}}{\sqrt{N}} \\
& c_{7}=\beta\left\{\frac{3 c_{1}{ }^{3} e^{3 \sqrt{N}}}{8 \sqrt{N}}-\frac{3 c_{2}{ }^{3} e^{-3 \sqrt{N}}}{8 \sqrt{N}}+\frac{3 c_{1}{ }^{2} c_{2}}{2 \sqrt{N}}\left(e^{\sqrt{N}}+\sqrt{N} e^{\sqrt{N}}\right)-\frac{3 c_{1} c_{2}{ }^{2}}{2 \sqrt{N}}\left(e^{-\sqrt{N}}-\sqrt{N} e^{-\sqrt{N}}\right)\right\}
\end{aligned}
$$

\section{HEAT TRANSFER ANALYSIS}

Now, considering the heat equation for the PTT fluid-model which takes care of heat conduction phenomena and energy loss due to viscous dissipation, we have neglected the joule-heating as the study is confined to low magnetic Reynolds number. Further, we have not considered Darcy dissipation term because our present flow field is not of extreme size or at extremely low temperature or high gravity field. In such situation, the Darcy dissipation term can be neglected while studying the flow through porous media.

Using boundary layer approximations, the heat transfer in the steady one dimensional flow of viscoelastic PTT fluid with boundary condition can be expressed as follows:

$$
k \frac{\partial^{2} T}{\partial y^{2}}+\tau_{x y} \frac{\partial u}{\partial y}+Q\left(T-T_{0}\right)=0
$$


$T(0)=T_{0}, T(L)=T_{1}$

Introducing the following nondimensional parameters in Equation (4.1)

$\theta=\frac{T-T_{0}}{T_{1}-T_{0}}, \operatorname{Pr}=\frac{\mu C_{p}}{k}, E c=\frac{U^{2}}{C_{p}\left(T_{1}-T_{0}\right)}, y^{*}=\frac{y}{L}, u^{*}=\frac{u}{U}, \tau^{*}=\frac{\tau L}{\mu U}, S=\frac{Q L^{2}}{k}$

we get (after dropping asterisk),

$\frac{\partial^{2} \theta}{\partial y^{2}}+P_{r} E_{c} \tau \frac{\partial u}{\partial y}+S \theta=0$

$\theta(0)=0, \theta(1)=1$

Substituting the values of $\tau_{x v}$ and $\mathrm{u}$ from (3.9) and integrating twice, we get the expression for $\theta$.

\section{RESULTS AND DISCUSSION}

The following discussion on temperature distribution reveals the effects of Deborah number and Eckert number which accounts for both momentum energy and thermal energy transport and resulting heating/cooling of the bounding surface besides other parameters on temperature field. However, the contribution of volumetric heat source has not been considered while computing to avoid the lengthy and complex calculations.

Figures 1-5 depict the effects of pertinent parameters on temperature distribution. The temperature distribution exhibits monotonically the increasing behavior across the flow domain. The effects of important parameters are as follows. The effect of the increase in magnetic parameter is to increase the temperature throughout the flow domain, but reverse effect is observed for all other parameters such as Deborah number $D_{e}$, porosity parameter $\mathrm{K}_{\mathrm{p}}$, Prandtl number $\mathrm{P}_{\mathrm{r}}$, and Eckert number $\mathrm{E}_{\mathrm{c}}$.

In the absence of viscous dissipation in the flow $\left(\mathrm{E}_{\mathrm{c}}=0\right)$, the temperature distribution is linear which is evident from energy Equation (4.3). The reason of the decrease in temperature with the increase in Pr is corroborative to the material property as Pr signifies the ratio of momentum diffusivity to thermal diffusivity. 


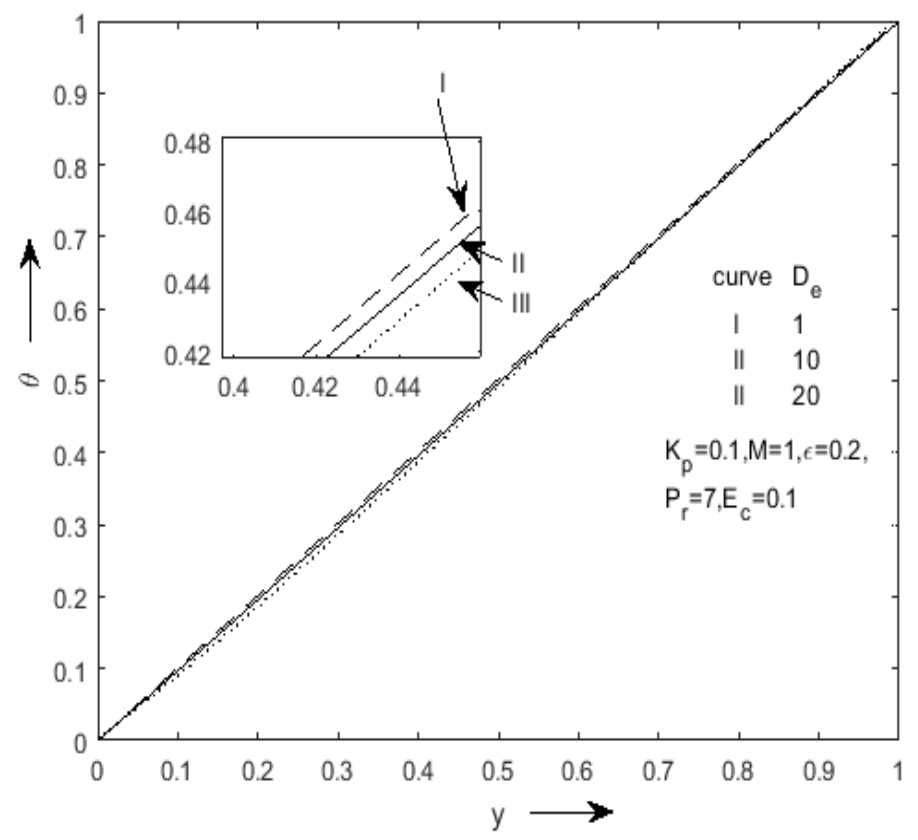

Figure 1. Temperature profiles for $D_{e}$.

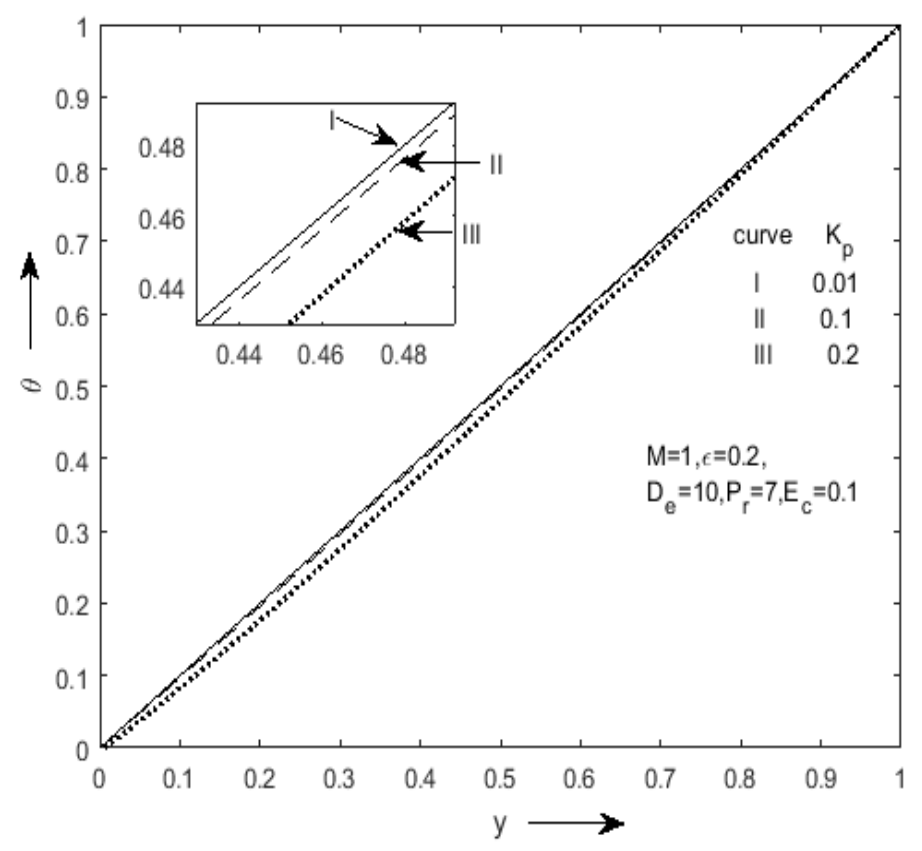

Figure 2. Temperature profiles for $\mathrm{Kp}$ 


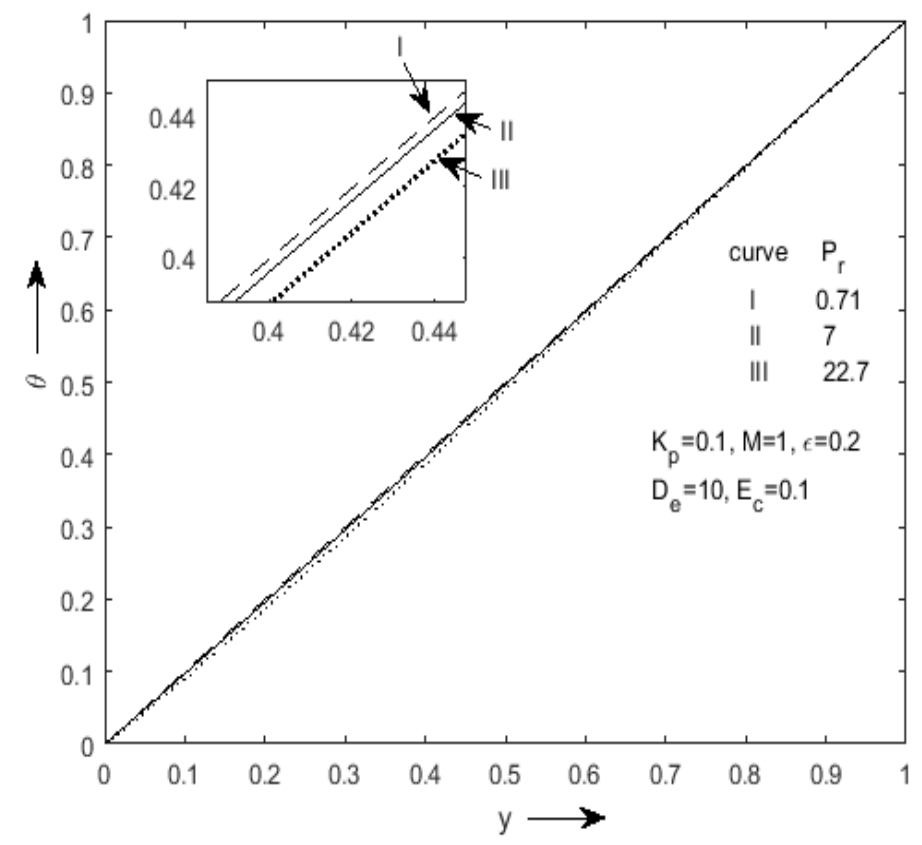

Figure 3. Temperature profiles for $\mathrm{P}_{\mathrm{r}}$

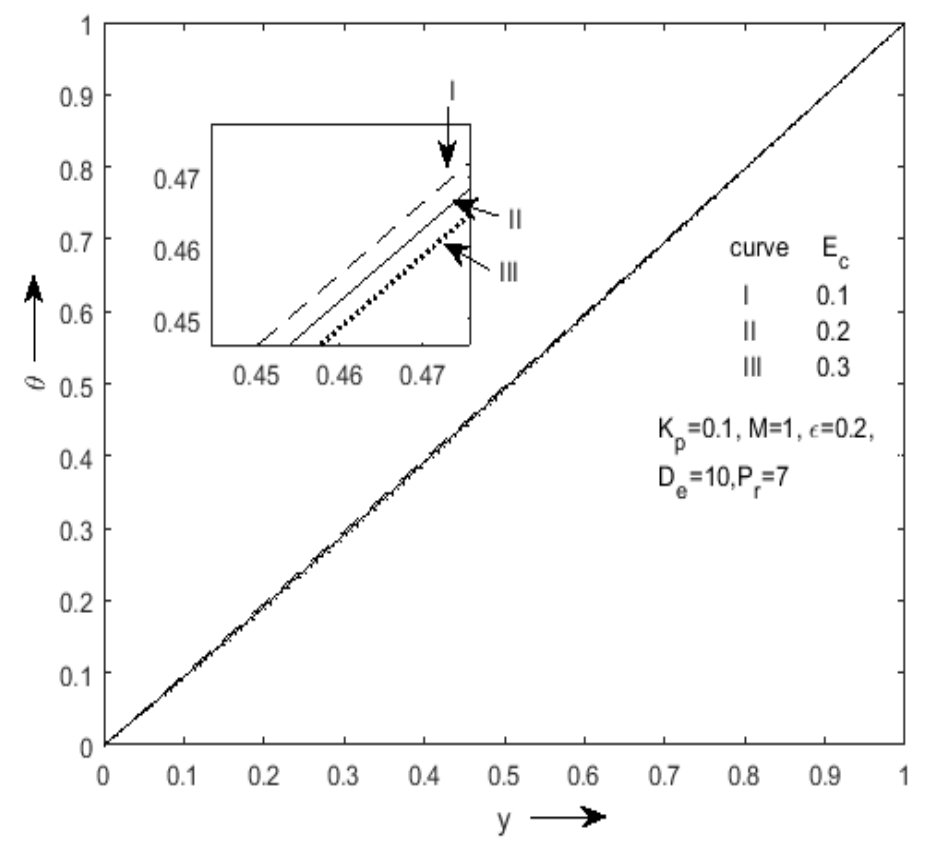

Figure 4. Temperature profiles for $E_{c}$ 


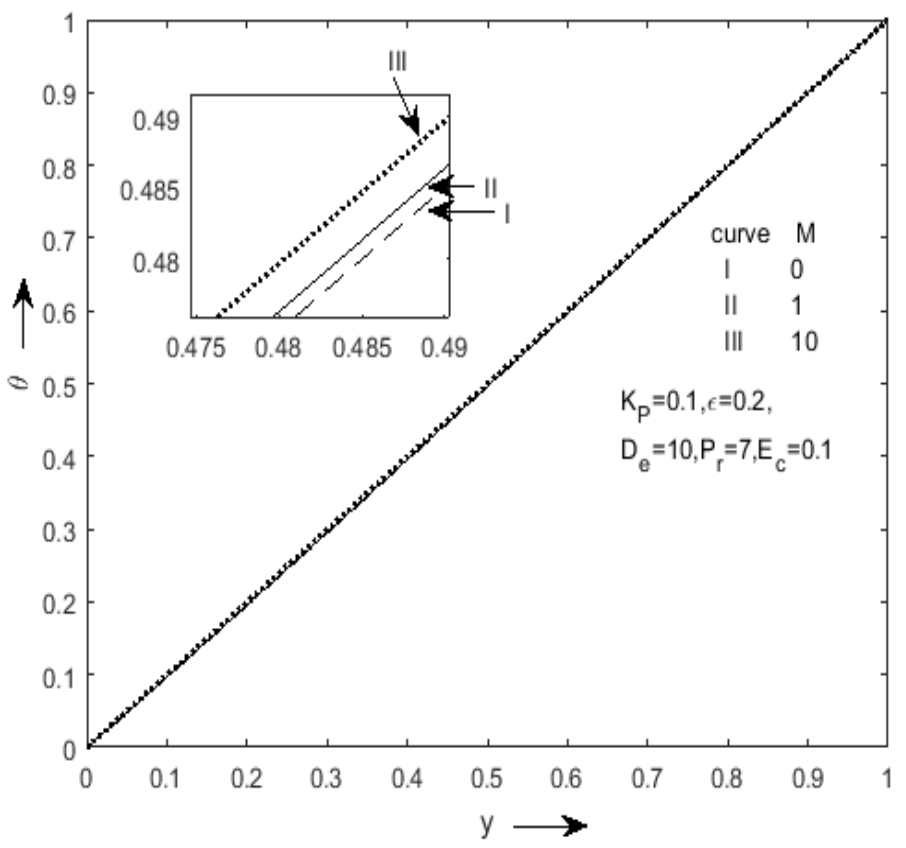

Figure 5. Temperature profiles for $M$.

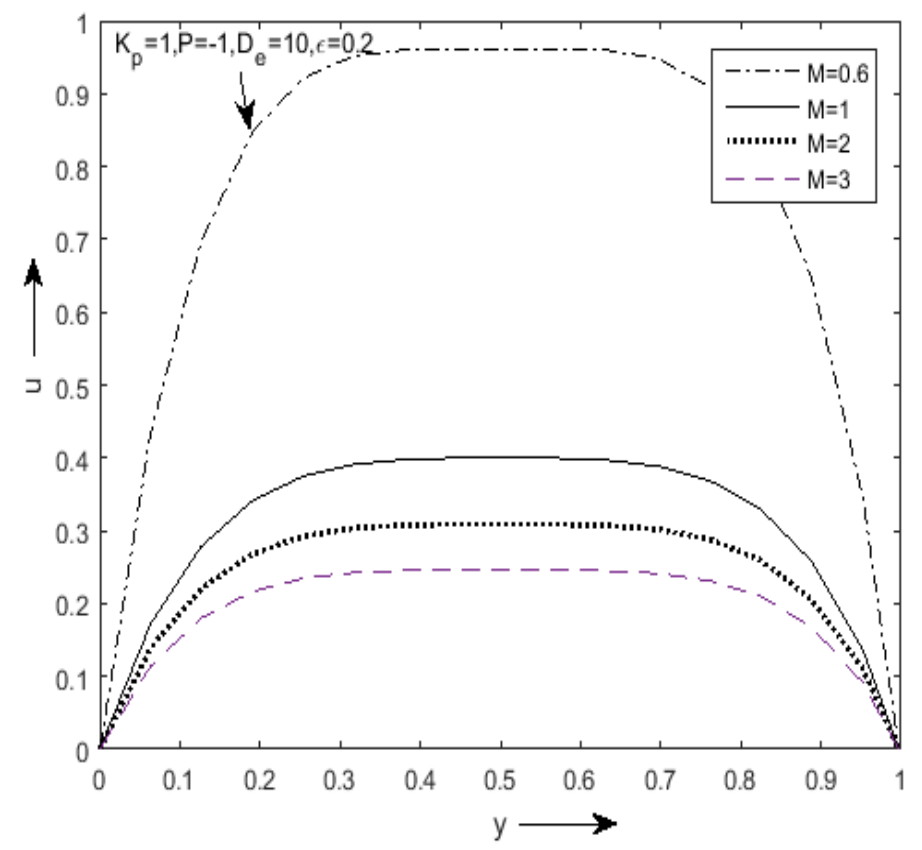

Figure 6. Velocity profiles for M. 
Fluid with higher $P_{r}$ will possess low conductivity, and hence temperature decreases as Pr increases. On the other hand, increase in magnetic force density parameter $\mathrm{M}$ generates force, which is proportional to negative of the velocity of the moving medium and acts as a viscous breaking force [12] and hence resists the motion and generates heat energy. Further, the effect of Deborah number which characterises both Newtonian $\left(\mathrm{D}_{\mathrm{e}}<1\right)$ and non-Newtonian elastic fluids $\left(D_{e}>1\right)$ is to decrease the temperature in the present study for both low and moderate values of $D_{e}$. This may be attributed to elastic property of the fluid for which some strain energy is stored up in the fluid mass, decreasing the temperature in the flow domain.

Fig. 6 illustrates the impact of electromagnetic force, a resistive electromagnetic force created due to interaction of transverse magnetic field with the conducting-flowing PTT fluid. Due to resistive force generated and acted upon in the main direction of flow, the velocity decreases. On careful observation, it is revealed that for low magnetic number vis-à-vis for low intensity of the applied magnetic field, the significant increase in velocity is marked. Therefore, during clinical/ mechanical necessity to control the flow of biological or industrial fluid flow, one can regulate the intensity of the external magnetic field to obtain the desired flow rate.

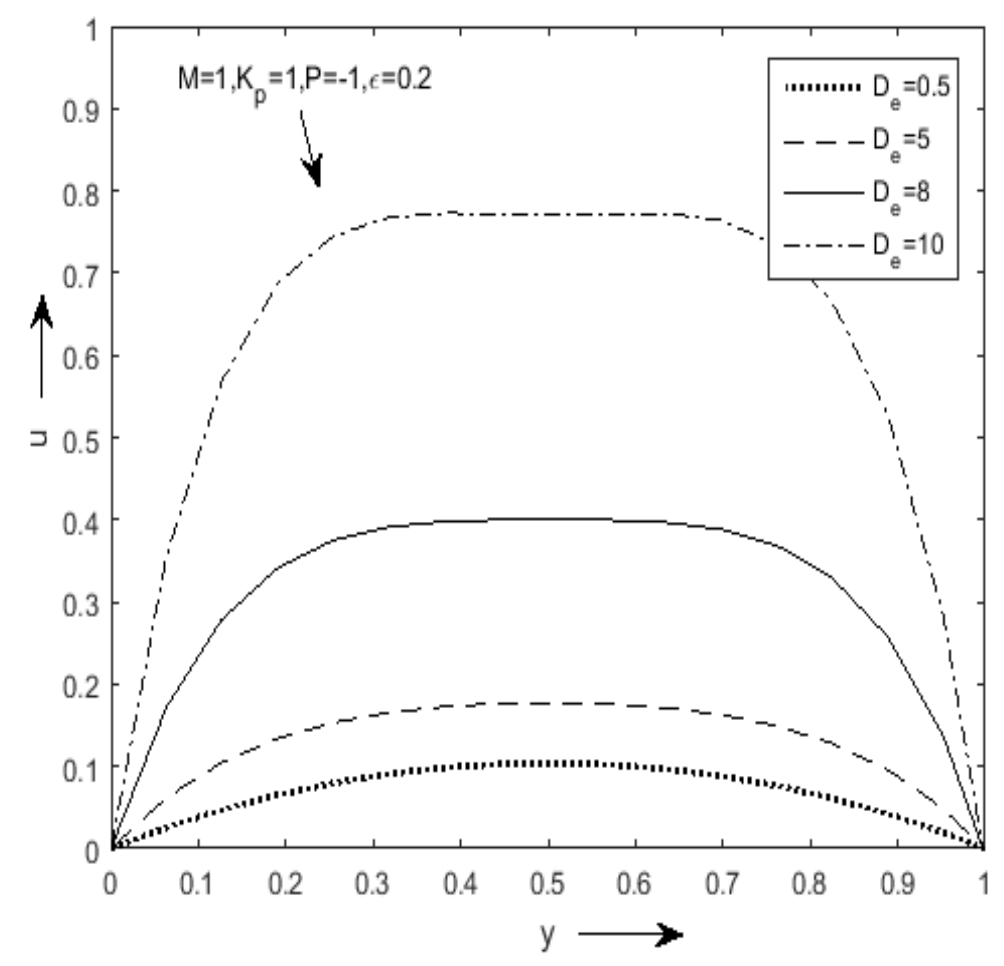

Figure 7. Velocity profiles for $\mathrm{D}_{\mathrm{e}}$ 


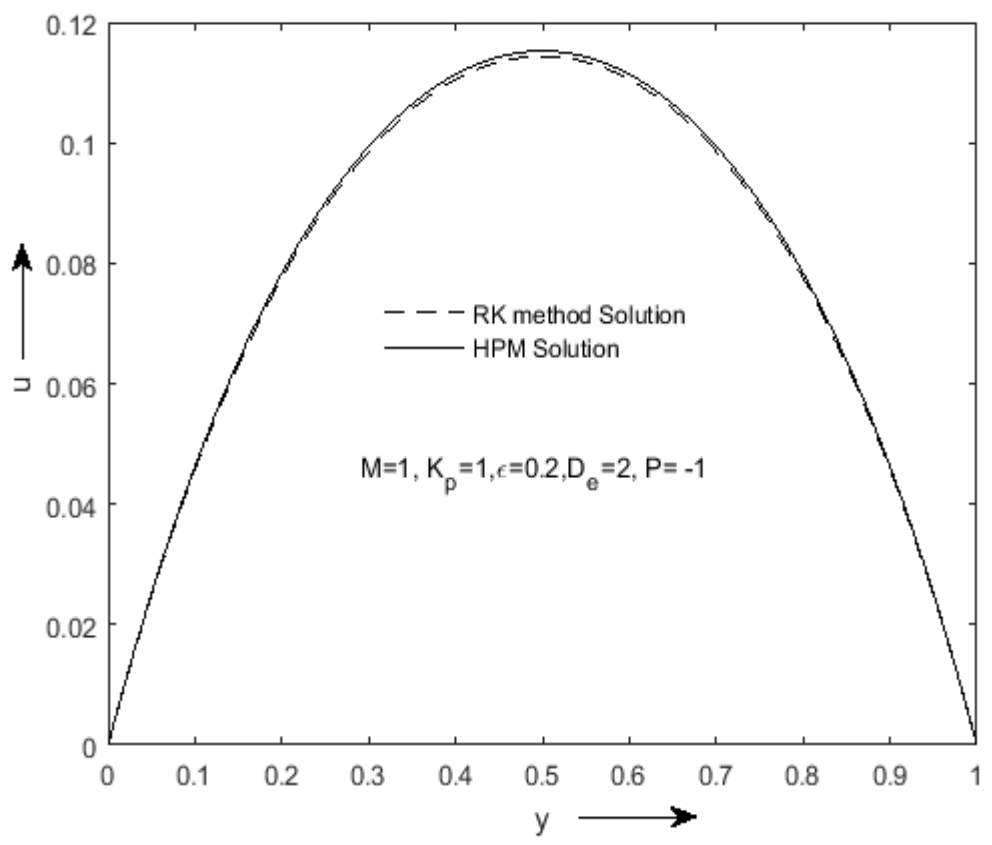

Figure 8 (a). Velocity profiles for $\mathrm{M}=1, \mathrm{Kp}=1$, and $\mathrm{De}=2$

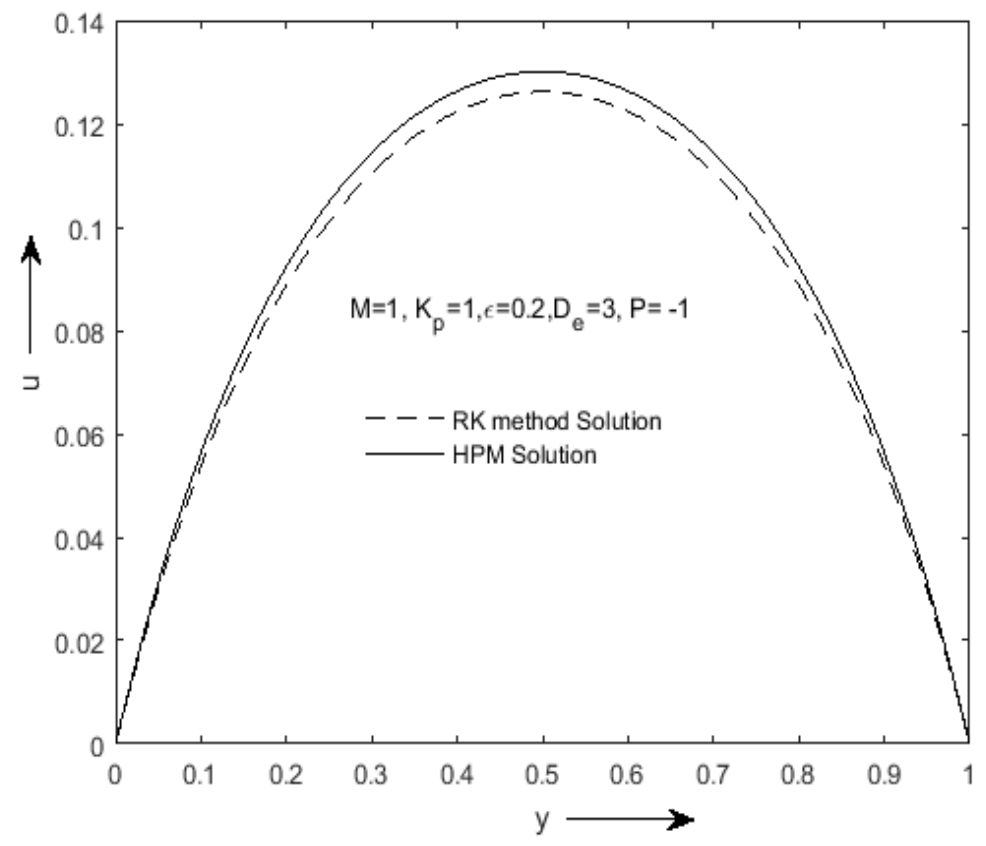

Figure 8(b). Velocity profiles for $D_{e}=3$ 


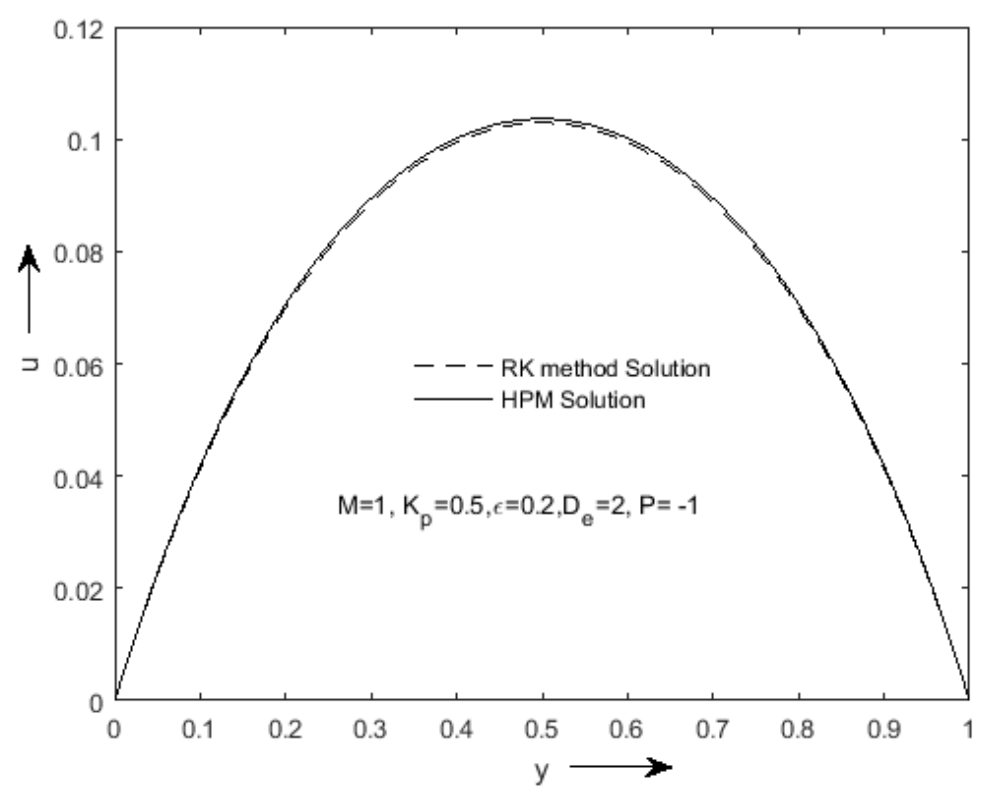

Figure 8(c). Velocity profiles for $\mathrm{K}_{\mathrm{p}}=0.5$

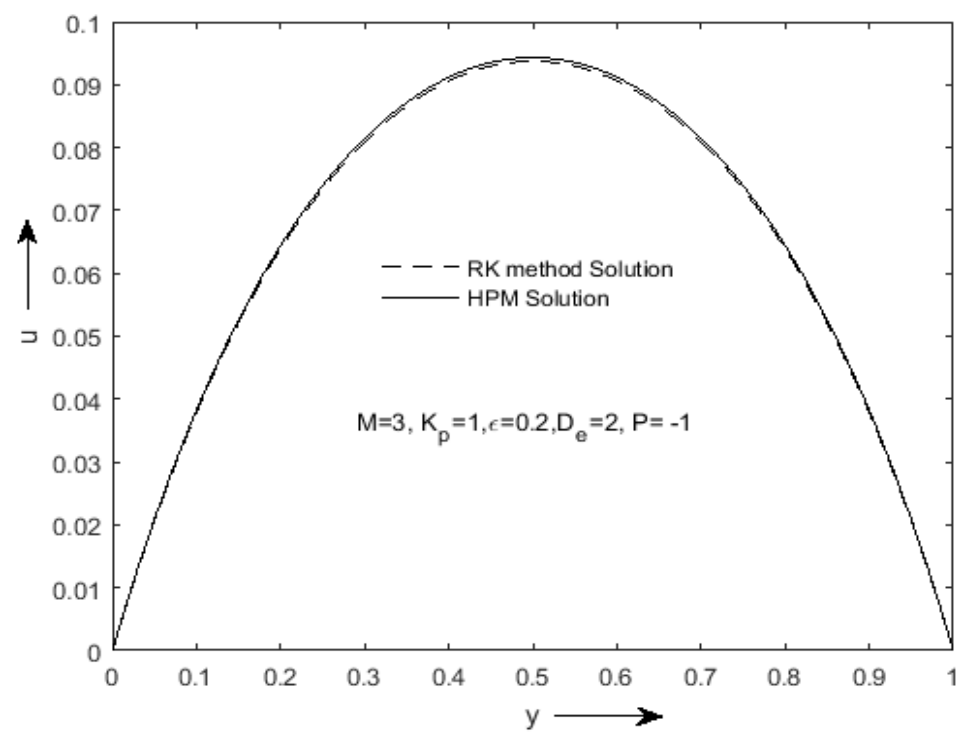

Figure 8(d). Velocity profiles for $M=3$

Figures 8(a)-8(d) are drawn to show the compatibility of two methods, that is, Runge-Kutta method and HPM in respect with different values of parameters $M, K_{p}$, and $D_{e}$. It is observed that both methods are in good agreement with each other except 8(b) for different values of $D_{e}$ where a slight difference is marked. The reason of difference may be attributed to the higher power of $\mathrm{D}_{\mathrm{e}}$, that is, $\mathrm{D}_{\mathrm{e}}{ }^{2}$ in Equation (2.20). For brevity, Figures 8(c) and $8(d)$ are omitted which correspond to different values of $M$ and $K_{p}$. 


\section{CONCLUSION}

The above discussion presents a flexible mean to simulate the heat transfer parameters to make use of P.T.T fluid as coolant or otherwise.

- Deborah number acts as a discriminating parameter between viscous and viscoelastic fluids in decelerating or accelerating the fluid velocity, respectively.

- Fluid with higher Pr possesses low conductivity, and hence temperature decreases.

- The temperature is decreased for both low and moderate values of Deborah number.

- Increase in magnetic force intensity parameter leads to increase in temperature and decrease in velocity.

- Temperature decreases for increasing values of both Eckert number and porosity parameter. Thus, higher porosity of the medium may act as a coolant.

- The almost linear variation of temperature distribution is the significant revelation of heat transfer for property of PTT flow ignoring thermal energy loss due to viscous dissipation (Equation. 4.3).

- Increase in magnetic force intensity increases the fluid temperature which may result in cooling of the bounding surface.

\section{ACKNOWLEDGEMENT}

The authors gratefully acknowledge the learned referee and editor in chief for constructive suggestions to complete recast and improve the manuscript.

\section{REFERENCES}

Nguyen, Q. D. \&Boger,D. V. 1998. Application of rheology to solving tailing disposal problems. International Journal of Mineral Processing 54(3):217-233.

Phan-Thien, N. \& Tanner, R.I. 1977. A New Constitutive Equation Derived fromNetwork Theory. Journal of Non-Newtonian Fluid Mechanics, 2, 353-365.

Tanner, R.I. 2000. Engineering Rheology. Clarendon Press, Oxford.

Oliveira, P.J. \&Pinho, F.T. 1999. Analytical Solution for the Fully-DevelopedChannel and Pipe Flow of PhanThien-Tanner Fluids. Journal of Fluid Mechanics, 387, 271-280.

Grillet, Anne M., Arjen, C.B.B., Gerrit, W.M. P. \& Frank, P.T. B. 2002. Stability analysis of constitutive equations for polymer melts in viscometric flows. J. Non-Newtonian Fluid Mech. 103, 221-250.

Oveisi, M. \&Abdollahzadeh, M. Y. 2016. Numerical study of slip effects on PTT fluids duct flow, Entomology and Applied Science Letters, 3, 5:148-168.

Shah, R. A., Ullah, M.\& Nasir J. 2017. Exact solution of non-isothermal PTT fluid in post treatment analysis of wire coating with slip boundary conditions. Sindh Univ. Res. Jour. (Sci. Ser.) 49(2), 425-432.

Ferras, L. L., Afonso, A. M., Alves, M. A., Nobrega, J. M.\&Pinho, F.T. 2020.Newtonian and viscoelastic fluid flows through an abrupt 1:4 expansion with slip boundary conditions, Phys. Fluids 32, 043103.

Song, D., Gupta, R. K.\&Chhabra, R. P. 2010. Effect of shear-thinning and elasticity in flow around a sphere in a cylindrical tube, Proceeding of the COMSOL conference, Boston.

Jamalabadi, M. Y. A. \&Oveisi, M.2016. Phan-Thien-Tanner modeling of a viscoelastic fluid flow around a cylinder in a duct, Journal of Chemical and Pharmaceutical Research. 8(1): 712-728. 
Hussain, S., Ali, N. \&Ullah K. 2019. Peristaltic flow of Phan-Thien-Tanner fluid: effects of peripheral layer and electro-osmotic force, Rheological Acta, 58, 603-618.

Khan, S. U. \& Tlili, I. 2020.Significance of activation energy and effective Prandtl number in accelerated flow of Jeffrey nanoparticles with gyrotactic microorganisms, Journal of Energy Resources Technology, 142(11), 112101-1.

Al-Khaled, K.\& Khan, S. U. 2020.Thermal aspects of Cassonnanoliquid with gyrotactic microorganisms, temperature dependent viscosity and variable thermal conductivity: Bio-technology and thermal applications, Inventions 5(3):39.

Ahmed, R., Ali, N., Khan, S. U. , Rashad, A. M., Nabwey, H. A. \& Tlili I. 2020 , Novel micro structural features on heat and mass transfer in peristaltic flow through a curved channel, Frontier in Physics, 8, 178 .

Khan, S. U., Al-Khaled, K.\&Khan, M. I., 2020.Convective nonlinear thermally developed flow of thixotropic nanoliquid configured by Riga surface with gyrotactic microorganism and activation energy: A biotechnology and thermal extrusion model, International Communications in Heat and Mass Transfer, 119, 104966.

Ahmed, R., Ali, N., Khan, S. U., Chamkha, A.\&Tlili I. 2020.Heat and mass transfer characteristics in flow of bi-viscosity fluid through a curved channel with contracting and expanding walls: A finite difference approach, Advances in Mechanical Engineering, 12(10) 1-16.

Dennis, G. Zill.2009, Differential Equation, Cengage Learning, 197.

He, J.H. 2005.Homotopy Perturbation Method for Bifurcation of Nonlinear Problems ,Int. J. Nonlin. Sci. Numer. Simul. 6(2),207-208. 